\title{
OP-0060 - Perceived barriers to leisure time physical activity in adults with type 2 diabetes attending primary health care in Oman: a cross-sectional survey
}

Thamra S Alghafri ${ }^{1}$, Saud M Alharthi ${ }^{1}$, Angela M Craigie ${ }^{2}$, Maureen Mcleod ${ }^{2}$, Annie S Anderson $^{2}$, Yahya Al-Farsi $^{3}$

1. Directorate General of Health Services, Ministry of Health, Muscat, Oman

2. Centre for Public Health Nutrition Research, University of Dundee, Ninewells Hospital and Medical School

3. Sultan Qaboos University, College of Medicine \& Health Sciences, Sultanate Of Oman

\section{Background}

Physical activity (PA) is fundamental in diabetes management for good metabolic control ${ }^{1}$. However, PA and diabetes are highly prevalent in the countries of the Gulf Cooperation Council ${ }^{2}$. This study aimed to identify barriers to performing leisure time PA and explore differences based on sociodemographic characteristics and perceived stages of change in PA in adults with type 2 diabetes (T2D) in Oman.

Methods

- Study design: A cross-sectional study using an Arabic version of the "Barriers to Being Active" 27 item questionnaire ${ }^{3}$ in 17 primary health centres randomly selected in Muscat.

- Population: Individuals $>18$ years with T2D, attending diabetes clinic for $>2$ years and with no contraindications to performing PA.

- Recruitment: All eligible patients attending their routine diabetes clinics during April and May 2015 were invited to participate.

- Ethics: Informed consent was obtained from participants.

- Information collected:

$\square$ Socio-demographic data.

$\square$ Perceptions on stage of change in $\mathrm{PA}^{4}$.

$\square$ Barriers to leisure PA. Participants rated on a scale of 0-9 how far factors under the following categories influenced their PA: fear of injury (FOI), lack of time (LOT), social support (LOSS), energy (LOE), willpower (LOW), skills (LOS), resources (LOR), religion (REL) and environment (ENV). Barriers were considered important if scored $\geq 5$.

- Analysis: Chi-square analysis (corrected for multiple analyses) was carried out to identify the distribution of the high barrier scores $(\geq 5)$ across the studied variables.

Results

The response rate was $98 \%$ (305/312).

Most $(96 \%)$ reported at least one barrier to leisure time PA.

L Lack of willpower (45.6\%), lack of resources (30.5\%), lack of social support $(29.2 \%)$ and fear of injury $(25.6 \%)$ were the most frequently reported barriers (Figure 1).

- Factors that differed by the studied variables:

$>$ Lack of willpower was reported significantly more often by individuals with low vs high income $(54.2 \%$ vs $40 \%, P=0.002)$ and in those reporting inactive vs active stages of change for PA $(50.7 \%$ vs $34.7 \%, P=0.029)$.

$>$ Lack of resources was reported significantly more often by those with low vs high income ( $40 \%$ vs $24.3 \%, P=0.004)$ and married vs unmarried $(33.8 \%$ vs $18.5 \%, P=0.018)$.

$>$ Lack of social support was reported significantly more often by females vs males (35.4\% vs $20.8 \%, P=0.005$ ) (Table 1 ).

\section{References}

1. ADA: Exercise and type 2 diabetes: ACSM and the ADA: Joint Position Statement. Diabetes Care 2010; 33(12): e147-e167

2. Alghafri TS et al., BMC Public Health 2017;18(1):85

3. AlQuaiz AM and Tayel SA. Ann Saudi Med 2009; 29(1):30-35

4. Martin SB et al.,Med Sci Sports Exerc 2000;32(12):2087-92
Table 1: P-values $(>0.05)$ * for distribution of high barrier scores $(\geq 5)$ to leisure physical activity

\begin{tabular}{|c|c|c|c|c|c|c|c|c|}
\hline LOT & LOSS & LOE & LOW & FOI & LOS & LOR & REL & ENV \\
\hline \multicolumn{9}{|c|}{ Gender: Male (42.6) vs Female (57.4) } \\
\hline $0.020 *$ & $0.008 *$ & 0.278 & 0.270 & 0.843 & $0.003^{*}$ & 0.640 & 0.981 & 0.508 \\
\hline \multicolumn{9}{|c|}{ Age: $<=57(50.8)$ vs $>57(49.2)$} \\
\hline $0.002 *$ & 0.347 & 0.232 & 1.0 & $0.008 *$ & $0.025 *$ & 0.292 & 1.0 & 0.942 \\
\hline \multicolumn{9}{|c|}{ Marital status: Unmarried (21.3) vs Married (78.7) } \\
\hline $0.047 *$ & 0.277 & 0.875 & 0.753 & 0.356 & 0.626 & $0.026 *$ & 0.231 & 0.720 \\
\hline \multicolumn{9}{|c|}{ Employment: Unemployed(76.6) vs Employed (23.3) } \\
\hline$<0.001 *$ & 0.120 & $0.003^{*}$ & 0.437 & $0.007 *$ & $0.004 *$ & 0.527 & 0.740 & 0.960 \\
\hline \multicolumn{9}{|c|}{ Education: Uneducated (48.9) vs Educated (51.1) } \\
\hline$<0.001 *$ & 0.129 & $0.042 *$ & 1.0 & $<0.001 *$ & $0.010 *$ & 0.816 & 0.162 & 0.700 \\
\hline \multicolumn{9}{|c|}{ Income: $<500(39.3)$ vs $>=500(60.7)$} \\
\hline 0.257 & 0.516 & 0.294 & $0.021 *$ & 0.163 & 0.663 & $0.005^{*}$ & 0.422 & 0.183 \\
\hline \multicolumn{9}{|c|}{ Self-reported stages of PA: Not active (67.9) vs Active (32.1) } \\
\hline 0.276 & 0.808 & 0.873 & $0.012 *$ & $<0.001 *$ & 0.161 & 0.667 & 1.0 & 0.244 \\
\hline
\end{tabular}

Environmental barriers $\quad \mathbf{1 1 . 8}$

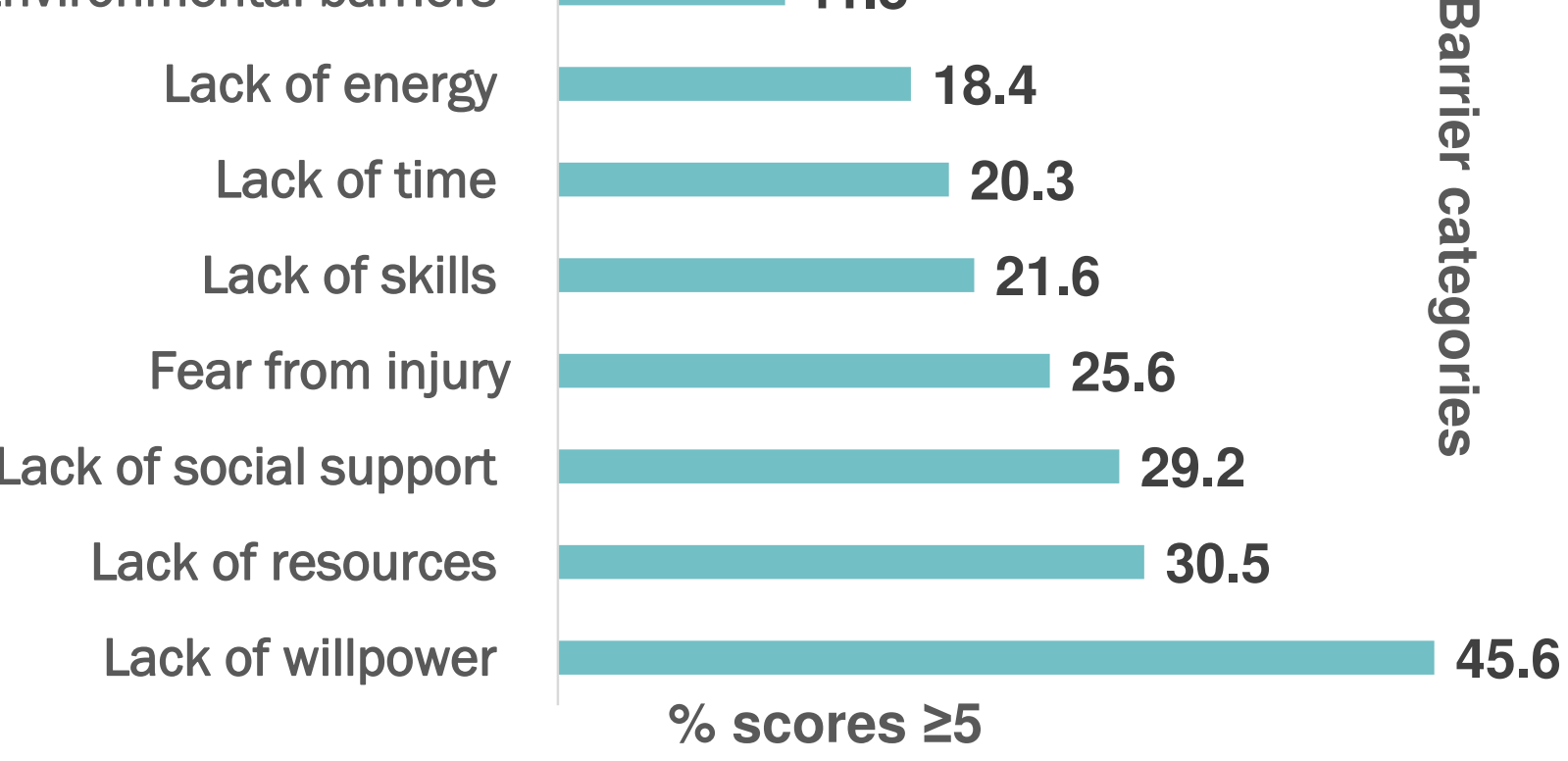

Figure 1: Percentage of population who reported high barrier scores $(\geq 5)$
Contact details:

Thamra S Alghafri: tsalghafri@dundee.ac.uk Saud M Alharthi: smsbar07@gmail.com

Yahya Al-farsi : ymfarsi@gmail.com

Elaine Bannerman: e.bannerman@dundee.ac.uk Angela M. Craigie: a.craigie@dundee.ac.uk

Annie S Anderson: a.s.anderson@dundee.ac.uk Copyright ( 2017

\section{Conclusion}

These findings suggest that physical activity interventions should efficacy and social support. 